\title{
Nonlinear Programming to Determine Best Weighted Coefficient of Balanced LINEX Loss Function Based on Lower Record Values
}

\author{
Fuad S. Al-Duais $\mathbb{D D}^{1,2}$ and Mohammed Alhagyan $\left.{ }^{1}\right)^{1}$ \\ ${ }^{1}$ Mathematics Department, College of Humanities and Science in Al Aflaj, Prince Sattam Bin Abdulaziz University, Al Aflaj, \\ Riyadh, Saudi Arabia \\ ${ }^{2}$ Administration Department, Administrative Science College, Thamar University, Thamar, Yemen
}

Correspondence should be addressed to Fuad S. Al-Duais; f.alduais@psau.edu.sa

Received 19 April 2021; Accepted 24 May 2021; Published 2 June 2021

Academic Editor: Ahmed Mostafa Khalil

Copyright (c) 2021 Fuad S. Al-Duais and Mohammed Alhagyan. This is an open access article distributed under the Creative Commons Attribution License, which permits unrestricted use, distribution, and reproduction in any medium, provided the original work is properly cited.

\begin{abstract}
Majority research studies in the literature determine the weighted coefficients of balanced loss function by suggesting some arbitrary values and then conducting comparison study to choose the best. However, this methodology is not efficient because there is no guarantee ensures that one of the chosen values is the best. This encouraged us to look for mathematical method that gives and guarantees the best values of the weighted coefficients. The proposed methodology in this research is to employ the nonlinear programming in determining the weighted coefficients of balanced loss function instead of the unguaranteed old methods. In this research, we consider two balanced loss functions including balanced square error (BSE) loss function and balanced linear exponential (BLINEX) loss function to estimate the parameter and reliability function of inverse Rayleigh distribution (IRD) based on lower record values. Comparisons are made between Bayesian estimators (SE, BSE, LINEX, and BLINEX) and maximum likelihood estimator via Monte Carlo simulation. The evaluation was done based on absolute bias and mean square errors. The outputs of the simulation showed that the balanced linear exponential (BLINEX) loss function has the best performance. Moreover, the simulation verified that the balanced loss functions are always better than corresponding loss function.
\end{abstract}

\section{Introduction}

Scholars always concern about how to find the best estimates of parameters and reliability function of the probability distributions. For this purpose, many methods were proposed. Some of these methods are classical, where they depend only on the sample information under study, assuming that the distribution parameter is fixed but unknown. There are other approaches (which are commonly known as Bayesian methods) that depend on merging prior information with sample information, assuming that the prior parameters behave as random variables, which are commonly known as prior probability distributions.

From a Bayesian perspective, the choice of the loss function is a crucial part of the estimation and prediction problems. To simplify the calculations, many authors prefer using a squared error loss function to produce Bayesian estimates. However, this loss function has mainly criticized where both of overestimation and underestimation are given equal importance, which does not agree with real practices. To deal with this situation, several asymmetric loss functions were proposed in the literature. For example, general entropy loss function (Abdel-Hamid [1]) and linear exponential (LINEX) loss function (Al-Duais and Alhagyan [2]; Khatun and Matin [3])

After that, the balanced loss function idea appeared in the literature which tried to reflect the desired criteria of two methods (see equation (13)), for example, balanced square error (BSE) loss function [4], balanced general entropy 
(BGE) loss function [5]), and balanced linear exponential (BLINEX) loss function [6] EL-Sagheer [7] EL-Sagheer [8].

However, the majority of proposed balanced loss functions in the literature determine the value of weighted coefficients $\omega_{1}$ and $\omega_{2}$ randomly without convinced mathematical justification. This motivated us to treat this issue by determining the weighted coefficients by using nonlinear programming. In this paper, we are going to use two balanced loss functions (i.e., BSE and BLINEX) to estimate the parameter and reliability function of inverse Rayleigh distribution (IRD) based on lower record values utilizing nonlinear programming in determining the best-weighted coefficients.

The IRD is considered as one of the important distributions. It has wide applications in the area of reliability theory, survival analysis, and life testing study. IRD under record value was studied by Muhammad [9]; Shawky and Badr [10]; Soliman et al. [11]; Manzoor et al. [12]; Rasheed and Aref [13]; and Abdullah and Aref [14].

The probability density function (pdf) and cumulative distribution function (cdf) of the IRD with scale parameter $\alpha$ are given, respectively, as follows:

$$
\begin{aligned}
& f(x ; \alpha)=\frac{2 \alpha}{x^{3}} \exp \left[-\frac{\alpha}{x^{2}}\right] ; \quad x>0, \alpha>0, \\
& F(x ; \alpha)=\exp \left[-\frac{\alpha}{x^{2}}\right] ; \quad x>0, \alpha>0 .
\end{aligned}
$$

Moreover, the reliability function $R(t)$ at mission time $t$ for the IRD is given by

$$
R(t ; \alpha)=1-\exp \left[-\frac{\alpha}{t^{2}}\right] ; \quad t>0
$$

\section{Record Values and Maximum Likelihood (ML) Estimation}

Let $X_{1}, X_{2}, X_{3}, \ldots$ be a sequence of independent and identically distributed (iid) random variables with (cdf) $F(x)$ and (pdf) $f(x)$. Set $Y_{m}=\min \left(X_{1}, X_{2}, X_{3}, \ldots, X_{m}\right), m \geq 1$. We say that $X_{j}$ is a lower record and denoted by $X_{L(i)}$ if $Y_{j}<Y_{j-1}, j>1$.

Assuming that $X_{L(1)}, X_{L(2)}, X_{L(3)}, \ldots X_{L(m)}$ are the first $m$ lower record values arising from a sequence $\left\{X_{i}\right\}$ of iid inverse Rayleigh distribution whose pdf and cdf are, respectively, given by (1) and (2). The joint density function of the first $m$ lower record values $\underline{x} \equiv x_{L(1)}, x_{L(2)}$, $x_{L(3)}, \ldots \ldots x_{L(m)}$ is given by

$$
f_{1,2,3, \ldots \ldots, n}\left(x_{L(1)}, x_{L(2)}, x_{L(3)}, \ldots \ldots x_{L(n)}\right)=f\left(x_{L(m)}\right) \prod_{i=1}^{m-1} \frac{f\left(x_{L(i)}\right)}{1-F\left(x_{L(i)}\right)}, \quad 0 \leq x_{L(1)}<x_{L(2)}<x_{L(3)}, \ldots \ldots<x_{L(m)}<\infty
$$

where $f($.$) and F($.$) are given, respectively, by (1) and (2)$ after replacing $x$ by $x_{L(i)}$. The likelihood function based on the $m$ lower record values $x$ is given by

$$
\mathscr{L}(\alpha \mid \underline{x})=(2 \alpha)^{m} u \exp \left[-\alpha T_{D}\right], \quad u=\prod_{i=1}^{m} x_{L(i)}^{-3}, T_{D}=x_{L(m)}^{-3}
$$

The log-likelihood function is written as

$$
\ln \mathscr{L}(\alpha \mid \underline{x})=m \ln (2 \alpha)-\alpha T_{D}-3 \sum_{i=1}^{m} \ln \left(x_{L(i)}\right) .
$$

By differentiating (6) with respect to the parameter $\alpha$ and equating to zero, the maximum likelihood estimate (MLE), under lower record value, say $\widehat{\alpha}_{\mathrm{ML}}$, was obtained as

$$
\widehat{\alpha}_{\mathrm{ML}}=\frac{m}{T_{D}} \text {. }
$$

By using the invariance property of the maximum likelihood estimator, the maximum likelihood estimator of reliability function $\widehat{R}(t)_{\mathrm{ML}}$ of $R(t)$ given by (3) after replacing $\alpha$ by $\widehat{\alpha}_{\mathrm{ML}}$ is

$$
\widehat{R}(t)_{\mathrm{ML}}=1-\exp \left[-\frac{\widehat{\alpha}_{\mathrm{ML}}}{t^{2}}\right], \quad t \geq 0
$$

\section{Loss Functions}

From a Bayesian perspective, the choice of loss function is an essential part in the estimation and prediction problems. In this work, we use three main types of loss function including squared error loss function, LINEX loss function, and balanced loss functions.

3.1. Squared Error (SE) Loss Function. SE loss function is a symmetric loss function. The SE loss function is expressed as follows:

$$
L(\widehat{\phi}, \phi)=(\widehat{\phi}-\phi)^{2}
$$

where $\widehat{\phi}$ is the estimation of parameter $\phi$. The Bayes estimator of $\phi$ based on SE loss function denoted by $\widehat{\phi}_{\mathrm{SE}}$ is obtained as follows:

$$
\widehat{\phi}_{\mathrm{SE}}=E_{\pi}(\widehat{\phi} \mid \underline{x})
$$


3.2. Linear Exponential (LINEX) Loss Function. LINEX loss function is an asymmetric loss function. The LINEX loss function is expressed as follows (see Varian [15]):

$$
L(\Delta) \propto[\exp [c \Delta]-c \Delta-1],
$$

where $\Delta=(\widehat{\phi}-\phi)$. The sign and magnitude of $c$ reflect the direction and degree of asymmetry, respectively. The Bayes estimator related to LINEX loss function, denoted by $\widehat{\phi}_{L}$, is given by

$$
\widehat{\phi}_{L}=-\frac{1}{c} \ln \left[E_{\phi}(\exp \{-c \phi\})\right], \quad c \neq 0,
$$

provided that $E_{\phi}(\exp \{-c \phi\})$ exists and finite, where $E_{\phi}$ denotes the expected value.

3.3. Balanced Loss Function (BLF). BLF is a mix of two estimators. In general, BLF is expressed as follows (see Jozani et al. [16]):

$$
\begin{aligned}
& L_{\rho, \omega, \gamma_{o}}(\hat{\phi}, \gamma)=\omega_{1} \rho\left(\phi, \gamma_{0}\right)+\omega_{2} \rho(\phi, \gamma) \omega_{1}+\omega_{2}=1, \\
& L_{\rho, \omega, \gamma_{o}}(\hat{\phi}, \gamma)=\omega_{1} \rho\left(\phi, \gamma_{0}\right)+\omega_{2} \rho(\phi, \gamma) \omega_{1}+\omega_{2}=1,
\end{aligned}
$$

where $\rho$ is an arbitrary loss function, while $\gamma_{0}$ is a chosen a prior target estimator of $\phi$ that can be obtained by several methods like maximum likelihood, least squares, or unbiasedness, and $\omega_{1}$ and $\omega_{2}$ represent weighted coefficient $\omega_{1}$ and $\omega_{2} \epsilon[0,1)$. In this work, we focus on two types of BLF, including balanced squared error (BSE) loss function and balanced LINEX (BLINEX) loss function.

3.3.1. Balanced Squared Error (BSE) Loss Function. BSE loss function is obtained by choosing $\rho(\phi, \gamma)=(\gamma-\phi)^{2}$, so equation (13) will be on the form (see Ahmadi et al. [17]):

$$
L_{\omega, \gamma_{0}}(\phi, \gamma)=\omega_{1}\left(\gamma-\gamma_{0}\right)^{2}+\omega_{2}(\gamma-\phi)^{2},
$$

and the corresponding Bayes estimate of the unknown parameter $\phi$ is given by

$$
\gamma_{\omega, \gamma_{0}}(\underline{x})=\omega_{1} \gamma_{0}+\omega_{2} E(\phi \mid \underline{x}) .
$$

Note that when $\omega_{1}=0$, then BSE loss function is just an SE loss function.

3.3.2. Balanced Linear Exponential (BLINEX) Loss Function. The BLINEX loss function is obtained by choosing $\rho(\phi, \gamma)=$ $\exp [-c(\phi-\gamma)-c(\phi-\gamma)-1]$ in equation (13) as follows (see Zellner [18]):

$$
\begin{aligned}
L_{\omega, \gamma_{0}}^{*}(\phi, \gamma)= & \omega_{1}\left[\exp \left[c\left(\gamma-\gamma_{0}\right)\right]-c\left(\gamma-\gamma_{0}\right)-1\right] \\
& +\omega_{2}[\exp [c(\gamma-\phi)]-c(\gamma-\phi)-1] .
\end{aligned}
$$

And the corresponding Bayes estimate of the unknown parameter $\phi$ is given by

$$
\gamma_{\omega, \gamma_{0}}^{*}(\underline{x})=-\frac{1}{c} \ln \left[\omega_{1} \exp \left[-c \gamma_{0}(\underline{x})\right]+\omega_{2} E(\exp (-c \phi \mid \underline{x}))\right] .
$$

It is worth noting, when $\omega_{1}=0$ then BLINEX loss function is just a LINEX loss function.

\section{Bayes Estimation}

In this section, we derive the Bayes estimates of the scale parameter $\alpha$ and the reliability $R(t)$ function of the IRD by using balanced loss functions (BLF). Furthermore, we assume gamma $(\varkappa, b)$ as a conjugate prior distribution for $\alpha$ as follows:

$$
g(\alpha)=\frac{b^{\varkappa}}{\Gamma(x)} \alpha^{\chi-1} \exp [-b \alpha] ; \quad b>0, \alpha>0 .
$$

By combining the likelihood function in equation (5) with the prior pdf of $\alpha$ in equation (18), we get the posterior distribution of $\alpha$ as

$$
\pi(\alpha \mid \underline{x})=\frac{L(\alpha ; \underline{x}) g(\alpha)}{\int_{0}^{\infty} L(\alpha ; \underline{x}) g(\alpha) \mathrm{d} \alpha}=\frac{\nu^{m+\varkappa}}{\Gamma(m+\varkappa)} \alpha^{m+\chi-1} \exp [-\alpha \nu],
$$

where

$$
\begin{aligned}
\underline{x} & \equiv x_{L(1)}, x_{L(2)}, x_{L(3)}, \ldots \ldots, x_{L(m)}, \\
\nu & =\left(b+T_{D}\right) .
\end{aligned}
$$

4.1. Estimates Based on Balanced Squared Error (BSE) Loss Function. Based on BSE loss function and by using equation (15), the Bayes estimate of a function $\phi$ where $\phi$ can be $\alpha$ or $R(t)$ is given by

$$
\phi_{\mathrm{BSE}}=\omega_{1} \widehat{\phi}_{\mathrm{ML}}+\omega_{2} E(\phi \mid \underline{x}),
$$

where $\widehat{\phi}_{\mathrm{ML}}$ is the ML estimate of $\phi$ and $E(\phi \mid \underline{x})$ can be obtained by

$$
E(\phi \mid \underline{x})=\int_{0}^{\infty} \phi \pi(\phi \mid \underline{x}) \mathrm{d} \phi .
$$

Based on the BSE loss function and by using equation (21), the Bayes estimator $\widehat{\alpha}_{\mathrm{BSE}}$ for $\alpha$ is

$$
\widehat{\alpha}_{\mathrm{BSE}}=\omega_{1} \widehat{\alpha}_{\mathrm{ML}}+\omega_{2} E(\alpha \mid \underline{x}),
$$

where $\widehat{\alpha}_{\mathrm{ML}}$ is the ML estimate of $\alpha$, which can be obtained using equation (7) and $E(\alpha \mid \underline{x})$ can be obtained using the following equation:

$$
E(\alpha \mid \underline{x})=\int_{0}^{\infty} \alpha \frac{\nu^{m+\chi}}{\Gamma(m+\chi)} \alpha^{m+\chi-1} \exp [-\alpha \nu] \mathrm{d} \alpha=\frac{\varkappa+m}{\nu} .
$$

Similarly, the Bayes estimate $\widehat{R}(t)_{\mathrm{BSE}}$ of the reliability $R(t)$ at a mission time $t$ related to BSE loss function is

$$
\widehat{R}(t)_{\mathrm{BSE}}=\omega_{1} \widehat{R}(t)_{\mathrm{ML}}+\omega_{2} E(R(t) \mid \underline{x}),
$$


where $\widehat{R}(t)_{\mathrm{ML}}$ is the ML estimate of $R(t)$ which can be obtained using equation (8) and $E(R(t) \mid \underline{x})$ can be obtained using the following equation:

$$
\begin{aligned}
E(R(t) \mid \underline{x}) & =\int_{0}^{\infty} 1-\exp \left[\frac{\alpha}{t^{2}}\right] \frac{\nu^{m+\varkappa}}{\Gamma(m+\varkappa)} \alpha^{m+\varkappa-1} \exp [-\alpha \nu] \mathrm{d} \alpha \\
& =1-\left(\frac{v}{\nu+t^{2}}\right)^{m+\varkappa} ; \quad t \geq .
\end{aligned}
$$

In this work, we solve the following nonlinear programming (using Mathematica software) to find the optimal values of the weighted coefficient $\omega_{1}$ and $\omega_{2}$ in equation (21):

$$
\begin{aligned}
& \text { minimize: } \operatorname{MSE}\left(\hat{\phi}_{\mathrm{BSE}}\right)=E\left(\hat{\phi}_{\mathrm{BSE}}-\phi\right)^{2} \\
&=E\left[\left(\omega_{1} \hat{\phi}_{\mathrm{ML}}+\omega_{2} E(\phi \mid \underline{x})-\phi\right)\right]^{2}
\end{aligned}
$$

subject to

$$
\begin{aligned}
& \omega_{1}+\omega_{2}=1, \\
& 0 \leq \omega_{1}<1, \\
& 0 \leq \omega_{2}<1 .
\end{aligned}
$$

4.2. Estimates Based on Balanced Linear Exponential (BLINEX) Loss Function. Based on BLINEX loss function and by using equation (16), the Bayes estimate of a function $\phi$ where $\phi$ can be $\alpha$ or $R(t)$ is given by

$$
\widehat{\phi}_{\mathrm{BL}}=-\frac{1}{c} \ln \left[\omega_{1} \exp \left[-c \widehat{\phi}_{\mathrm{ML}}\right]+\omega_{2} E(\exp [-c \phi] \mid \underline{x})\right],
$$

where $\widehat{\phi}_{\mathrm{ML}}$ is the ML estimate of $\phi$ and $E(\exp [-c \phi] \mid \underline{x})$ can be obtained by

$$
E(\exp [-c \phi] \mid \underline{x})=\int_{0}^{\infty} \exp [(-c \phi \mid \underline{x})] \pi(\phi \mid \underline{x}) \mathrm{d} \phi .
$$

Based on BLINEX loss function and by using equation (28), the Bayes estimator $\widehat{\alpha}_{\mathrm{BL}}$ for $\alpha$ is given as

$$
\widehat{\alpha}_{\mathrm{BL}}=-\frac{1}{c} \ln \left[\omega_{1} \exp \left[-c \widehat{\alpha}_{\mathrm{ML}}\right]+\omega_{2} E(\exp [-c \alpha] \mid \underline{x})\right],
$$

where $\widehat{\alpha}_{\mathrm{ML}}$ is the ML estimate of $\alpha$ which can be obtained using equation (7) and $E(\exp [-c \alpha] \mid \underline{x})$ can be obtained using the following integral:

$$
\begin{aligned}
E(\exp [-c \alpha] \mid \underline{x})= & \int_{0}^{\infty} \exp [(-c \alpha \mid \underline{x})] \pi(\alpha \mid \underline{x}) \mathrm{d} \alpha \\
= & \int_{0}^{\infty} \exp [-c \alpha] \frac{\nu^{m+\varkappa}}{\Gamma(m+\chi)} \alpha^{m+\chi-1} \exp \\
& \cdot[-\alpha v] \mathrm{d} \alpha \\
= & \left(1+\frac{c}{v}\right)^{-(m+\chi)} .
\end{aligned}
$$

Similarly, the Bayes estimate $\widehat{R}(t)_{\mathrm{BL}}$ of the reliability $R(t)$ at a mission time $t$ related to BLINEX loss function is

$$
\widehat{R}(t)_{\mathrm{BL}}=-\frac{1}{c} \ln \left[\omega_{1} \exp \left[-c \widehat{R}(t)_{\mathrm{ML}}\right]+\omega_{2} E(\exp [-c R(t)] \mid \underline{x})\right],
$$

where $\widehat{R}(t)_{\mathrm{ML}}$ is the ML estimate of $R(t)$ which can be obtained using equation (8) and $E(\exp [-c R(t)] \mid \underline{x})$ can be obtained using the following integral:

$$
\begin{aligned}
E(\exp [-c R(t)] \mid \underline{x})= & \int_{0}^{\infty} \exp [(-c R(t) \mid \underline{x})] \pi(\alpha \mid \underline{x}) \mathrm{d} \alpha \\
= & \int_{0}^{\infty} \exp \left[-c\left(1-\exp \left[-\frac{\alpha}{t^{2}}\right]\right)\right] \\
& \frac{v^{m+\varkappa}}{\Gamma(m+x)^{m+\chi-1}} \exp [-\alpha v] \mathrm{d} \alpha \\
= & \exp [-c]+\exp [-c] \nu^{m+x} \sum_{i=1}^{n} \frac{(c)^{i}}{i !} \\
& \cdot\left(\nu+i t^{-2}\right)^{-(m+x)} .
\end{aligned}
$$

In this work, we solve the following nonlinear programming (using Mathematica software) to find the optimal values of the weighted coefficient $\omega_{1}$ and $\omega_{2}$ in equation (28):

minimize: $\operatorname{MSE}\left(\widehat{\phi}_{\mathrm{BL}}\right)=E\left(\widehat{\phi}_{B l}-\phi\right)^{2}$

$$
=\left[-\frac{1}{c} \ln \left[\omega_{1} \exp \left[-c \widehat{\phi}_{\mathrm{ML}}\right]+\omega_{2} E(\exp [-c \phi] \mid \underline{x})\right]-\phi\right]^{2}
$$

subject to

$$
\begin{aligned}
& \omega_{1}+\omega_{2}=1, \\
& 0 \leq \omega_{1}<1 \\
& 0 \leq \omega_{2}<1 \\
& \widehat{\phi}_{\mathrm{BL}}=-\frac{1}{c} \ln \left[\omega_{1} \exp \left[-c \widehat{\phi}_{\mathrm{ML}}\right]+\omega_{2} E(\exp [-c \phi] \mid \underline{x})\right] .
\end{aligned}
$$

\section{Simulation Study and Comparisons}

All estimation methods, mentioned in Section 4, are used to estimate the parameter and reliability function of IRD. To examine the performance of these estimation methods, the Monte Carlo simulation study is conducted. The simulation consists of four steps as follows:

(1) For the given values of prior parameters ( $b=2, x=1)$, generate a random value $\alpha=1.383$ from the Gamma prior pdf in equation (18) [19]. 
TABLE 1: Absolute bias of the estimates of $\alpha$

\begin{tabular}{|c|c|c|c|c|c|c|c|c|c|}
\hline \multirow{2}{*}{$\mathbf{m}$} & \multirow{2}{*}{$\widehat{\alpha}_{\mathrm{ML}}$} & \multirow{2}{*}{$\widehat{\alpha}_{\mathrm{SE}}$} & \multirow{2}{*}{$\widehat{\alpha}_{\text {BSE }}$} & \multicolumn{3}{|c|}{$\widehat{\alpha}_{L}$} & \multicolumn{3}{|c|}{$\widehat{\alpha}_{\mathrm{BL}}$} \\
\hline & & & & $c=0.001$ & $c=1$ & $c=2$ & $c=0.001$ & $c=1$ & $c=2$ \\
\hline 3 & 1.03787 & 0.60693 & 0.56808 & 0.60662 & 0.39863 & 0.31514 & 0.56787 & 0.39849 & 0.28874 \\
\hline 4 & 0.78828 & 0.55394 & 0.51968 & 0.55372 & 0.39272 & 0.32124 & 0.51954 & 0.39257 & 0.29764 \\
\hline 5 & 0.65342 & 0.50596 & 0.47534 & 0.50578 & 0.37810 & 0.31704 & 0.47525 & 0.37793 & 0.29541 \\
\hline 6 & 0.55881 & 0.46009 & 0.43195 & 0.45996 & 0.35661 & 0.30454 & 0.43188 & 0.35644 & 0.28420 \\
\hline 7 & 0.48079 & 0.41094 & 0.38500 & 0.41083 & 0.32850 & 0.28677 & 0.38495 & 0.32832 & 0.26636 \\
\hline
\end{tabular}

TABle 2: MSEs of the estimates of $\alpha$

\begin{tabular}{|c|c|c|c|c|c|c|c|c|c|}
\hline \multirow{2}{*}{ m } & \multirow{2}{*}{$\widehat{\alpha}_{\mathrm{ML}}$} & \multirow{2}{*}{$\widehat{\alpha}_{\mathrm{SE}}$} & \multirow{2}{*}{$\widehat{\alpha}_{\text {BSE }}$} & \multicolumn{3}{|c|}{$\widehat{\alpha}_{\mathrm{L}}$} & \multicolumn{3}{|c|}{$\widehat{\alpha}_{\mathrm{BL}}$} \\
\hline & & & & $c=0.001$ & $c=1$ & $c=2$ & $c=0.001$ & $c=1$ & $c=2$ \\
\hline 3 & 4.55002 & 0.66780 & 0.65187 & 0.66706 & 0.26109 & 0.14830 & 0.65120 & 0.26109 & 0.14235 \\
\hline 4 & 1.82889 & 0.58323 & 0.58270 & 0.58270 & 0.26526 & 0.15928 & 0.56883 & 0.26526 & 0.15391 \\
\hline 5 & 1.13061 & 0.49605 & 0.48381 & 0.49566 & 0.25113 & 0.15827 & 0.48348 & 0.25113 & 0.15330 \\
\hline 6 & 0.77355 & 0.41691 & 0.40581 & 0.41662 & 0.22785 & 0.14891 & 0.40557 & 0.22785 & 0.14420 \\
\hline 7 & 0.54374 & 0.34428 & 0.33437 & 0.34407 & 0.19897 & 0.13374 & 0.33420 & 0.19897 & 0.12880 \\
\hline
\end{tabular}

TABLE 3: Absolute bias of the estimates of $R(t)$ at $t=4$

\begin{tabular}{|c|c|c|c|c|c|c|c|c|c|}
\hline \multirow{2}{*}{ m } & \multirow{2}{*}{$\widehat{R}(\mathbf{t})_{\mathrm{ML}}$} & \multirow{2}{*}{$\widehat{R}(\mathbf{t})_{\mathrm{SE}}$} & \multirow{2}{*}{$\widehat{R}(\mathbf{t})_{\mathrm{BSE}}$} & \multicolumn{3}{|c|}{$\widehat{R}(\mathbf{t})_{\mathbf{L}}$} & \multicolumn{3}{|c|}{$\widehat{R}(\mathbf{t})_{\mathrm{BL}}$} \\
\hline & & & & $c=-2$ & $c=0.001$ & $c=2$ & $c=-2$ & $c=0.001$ & $c=2$ \\
\hline 3 & 0.05343 & 0.03276 & 0.03090 & 0.03444 & 0.03276 & 0.03120 & 0.03195 & 0.03090 & 0.02985 \\
\hline 4 & 0.04251 & 0.03013 & 0.02848 & 0.03137 & 0.03013 & 0.02896 & 0.02918 & 0.02848 & 0.02777 \\
\hline 5 & 0.03582 & 0.02769 & 0.02622 & 0.02866 & 0.02769 & 0.02677 & 0.02670 & 0.02621 & 0.02571 \\
\hline 6 & 0.03093 & 0.02529 & 0.02394 & 0.03444 & 0.03276 & 0.03120 & 0.03195 & 0.03090 & 0.02985 \\
\hline 7 & 0.02678 & 0.02267 & 0.02142 & 0.03137 & 0.03013 & 0.02896 & 0.02918 & 0.02848 & 0.02777 \\
\hline
\end{tabular}

TABLE 4: MSEs of the estimates of $R(t)$ at $t=4$

\begin{tabular}{|c|c|c|c|c|c|c|c|c|c|}
\hline \multirow{2}{*}{ m } & \multirow{2}{*}{$\widehat{R}(\mathbf{t})_{\mathrm{ML}}$} & \multirow{2}{*}{$\widehat{R}(\mathbf{t})_{\mathrm{SE}}$} & \multirow{2}{*}{$\widehat{R}(\mathbf{t})_{\mathrm{BSE}}$} & \multicolumn{3}{|c|}{$\widehat{R}(\mathbf{t})_{\mathbf{L}}$} & \multicolumn{3}{|c|}{$\widehat{R}(\mathbf{t})_{\mathrm{BL}}$} \\
\hline & & & & $c=-2$ & $c=0.001$ & $c=2$ & $c=-2$ & $c=0.001$ & $c=2$ \\
\hline 3 & 0.00815 & 0.00188 & 0.00184 & 0.00209 & 0.00188 & 0.00209 & 0.00203 & 0.00184 & 0.00167 \\
\hline 4 & 0.00457 & 0.00166 & 0.00163 & 0.00181 & 0.00166 & 0.00153 & 0.00176 & 0.00163 & 0.00151 \\
\hline 5 & 0.00304 & 0.00143 & 0.00140 & 0.00154 & 0.00143 & 0.00133 & 0.00149 & 0.00140 & 0.00131 \\
\hline 6 & 0.00216 & 0.00121 & 0.00119 & 0.00130 & 0.00121 & 0.00114 & 0.00126 & 0.00119 & 0.00112 \\
\hline 7 & 0.00158 & 0.00101 & 0.00099 & 0.00107 & 0.00101 & 0.00095 & 0.00104 & 0.00099 & 0.00094 \\
\hline
\end{tabular}

(2) Using $\alpha$ obtained in Step 1, we generatem $(m=3,4,5,6,7)$ lower record values from inverse Rayleigh distribution whose pdf is given by equation (1).

(3) The different estimates of $\alpha$ and $R(t)$ at time $t$ (chosen to be 4) are computed.

(4) Steps 1 to 3 are repeated 10,000 times.

(5) The evaluation is done depending on the absolute bias in addition to the mean square error (MSE)

$$
\begin{aligned}
\text { absolute bias }(\widehat{\phi}) & =\frac{1}{10000} \sum_{i=1}^{10000}|\widehat{\phi}-\phi|, \\
\operatorname{MSE}(\widehat{\phi}) & =\frac{1}{10000} \sum_{i=1}^{10000}(\widehat{\phi}-\phi)^{2},
\end{aligned}
$$

where $\widehat{\phi}$ is the estimate at the $i^{\text {th }}$ run.

(6) The results are listed in Tables 1-4. 


\section{Concluding Remarks}

In this paper, nonlinear programming was employed to get the best values of weighted coefficients $\left(\omega_{1}\right.$ and $\left.\omega_{2}\right)$ of the balanced loss function. The Bayesian and non-Bayesian estimates of the parameter $\alpha$ and reliability function $R(t)$ of the lifetimes follow the inverse Rayleigh distribution. The estimations were conducted depending on lower record values.

The results are listed in Tables 1-4. The main observations are stated in the following points:

(1) All tables showed that the Bayes estimates under BLINEX loss function are the best according to the smallest values of absolute bias and MSE comparing with the estimates under LINEX loss function, BSE loss function, SE loss function, or MLEs. Bayes estimates under the BSE loss function came in the second level of accuracy. The third, fourth, and fifth levels of accuracy were for Bayes estimates under the LINEX loss function, the estimates under SE loss function, and ML estimations, respectively.

(2) The results showed that the values of all MSEs and all absolute bias decrease as $m$ increases. This means here is an inverse relationship between the evaluation functions and the number of recorded values.

(3) In order to show the effect of the shape parameter of the asymmetric loss function, we examined different values of $c$. One can observe that when the value of $c$ is closed to zero, then the values of MSE of both Bayes estimates under LINEX and BLINEX loss function are almost the same. This means that BLINEX loss function is generated to LINEX loss function.

\section{Data Availability}

The data were generated by simulation done by using mathematical software.

\section{Conflicts of Interest}

The authors declare that they have no conflicts of interest.

\section{References}

[1] A. H. Abdel-Hamid, "Estimations in step-partially accelerated life tests for an exponential lifetime model under progressive type-I censoring and general entropy loss function," Journal of Mathematics and Statistical Science, pp. 75-93, 2016, p.

[2] F. Al-Duais and M. Alhagyan, "Bayesian estimates based on record values under weighted LINEX loss function," Pakistan Journal of Statistics and Operation Research, vol. 16, no. 1, pp. 11-19, 2020, p.

[3] N. Khatun and M. A. Matin, "A study on LINEX loss function with different estimating methods," Open Journal of Statistics, vol. 10, no. 01, pp. 52-63, 2020.

[4] M. V. Ahmadi and M Doostparast, "Pareto analysis for the lifetime performance index of products on the basis of progressively first-failure-censored batches under balanced symmetric and asymmetric loss functions," Journal of Applied Statistics, vol. 46, no. 7, pp. 1196-1227, 2019.

[5] R. Xu and W. Gui, "Entropy estimation of inverse Weibull distribution under adaptive type-II progressive hybrid censoring schemes," Symmetry, vol. 11, no. 12, p. 1463, 2019.

[6] A. H. A. Ellah, "Bayesian and non-Bayesian estimation of the inverse Weibull model based on generalized order statistics," vol. 4b, 2012.

[7] R. M. EL-Sagheer, "Estimation of parameters of WeibullGamma distribution based on progressively censored data," Statistical Papers, vol. 59, no. 2, pp. 725-757, 2018.

[8] R. M. El-Sagheer, "Estimation of the parameters of life for distributions having power hazard function based on progressively Type-II censored data," Advances and Applications in Statistics, vol. 45, no. 1, pp. 1-27, 2015, p.

[9] M. Muhammad, "Some distributional properties and estimation of parameters for inverse Rayleigh distribution through lower record values," International Journal of Statistics and Systems, vol. 2, no. 1, pp. 15-19, 2007, p.

[10] A. I. Shawky and M. M. Badr, "Estimations and prediction from the inverse Rayleigh model based on lower record statistics," Life Science Journal, vol. 9, no. 2, pp. 985-990, 2012, p.

[11] A. Soliman, E. A. Amin, and A. A. Abd-El Aziz, "Estimation and prediction from inverse Rayleigh distribution based on lower record values," Applied Mathematical Sciences, vol. 4, no. 62 , pp. 3057-3066, 2010, p.

[12] S. Manzoor, A. Z. Memon, M. Firdos, T. Kanwal, and R. Manzoor, "Estimation of inverse Rayleigh distribution parameters for type II singly and doubly censored data based on lower record values," International Journal of Probability and Statistics, vol. 7, no. 1, pp. 1-13, 2018, p.

[13] H. A. Rasheed and R. K. Aref, "Bayes estimator for inverse Rayleigh distribution under generalized weighted loss function," Mathematics and Statistics Journal, vol. 3, no. 1, pp. 18-24, 2017, p.

[14] H. Abdullah and R. Aref, "Bayesian approach in estimation of scale parameter of inverse Rayleigh distribution," Mathematics and Statistics Journal, vol. 2, no. 1, pp. 8-13, 2016, p.

[15] H. R. Varian, "A Bayesian approach to real estate assessment," Studies in Bayesian Econometric and Statistics in Honor of Leonard J. Savage, pp. 195-208, North-Holland Publishing Co., Amsterdam, Netherlands, 1975.

[16] M. Jafari Jozani, É. Marchand, and A. Parsian, "Bayesian and Robust Bayesian analysis under a general class of balanced loss functions," Statistical Papers, vol. 53, no. 1, pp. 51-60, 2012, p.

[17] J. Ahmadi, M. Jafari Jozani, É. Marchand, and A. Parsian, "Bayes estimation based on-record data from a general class of distributions under balanced type loss functions," Journal of Statistical Planning and Inference, vol. 139, no. 3, pp. 11801189, 2009, p.

[18] A. Zellner, "Bayesian estimation and prediction using asymmetric loss functions," Journal of the American Statistical Association, vol. 81, no. 394, pp. 446-451, 1986, p.

[19] K. S. Sultan, "Bayesian estimates based on record values from the inverse Weibull lifetime model," Quality Technology \& Quantitative Management, vol. 5, no. 4, pp. 363-374, 2008, p. 